\title{
Las comunidades seguras: una sinopsis
}

\author{
Andrés Fandiño-Losada, MD, MSc, ${ }^{(1)}$ Shrikant I Bangdiwala, $\mathrm{PhD},{ }^{(2)}$ \\ María Isabel Gutiérrez, MD, MSc, PhD, (3) Leif Svanström, BA, MD, PhD. (4)
}

Fandiño-Losada A, Bangdiwala SI, Gutiérrez MI, Svanström L. Las comunidades seguras: una sinopsis. Salud Publica Mex 2008;50 supl I:S78-S85.

\section{Resumen}

La comunidad segura es un modelo de intervención de la Organización Mundial de la Salud (OMS) para el control y la prevención de las lesiones y la violencia basada en la comunidad. El programa está dirigido a la prevención de todos los tipos de lesiones y todos los grupos de edad, ambientes y situaciones. En este modelo, la comunidad identifica sus necesidades organizativas, define las áreas de interés con la participación de todos, diseña e instituye las intervenciones por medio de un trabajo intersectorial y ofrece soluciones locales de bajo costo; asimismo, garantiza la sostenibilidad de los programas mediante el compromiso gubernamental y las alianzas entre diferentes organizaciones. Este modelo ha demostrado su efectividad en diferentes contextos, incluidos los países en desarrollo. En Latinoamérica se ha certificado una "comunidad segura" en Chile, un ejemplo a seguir para enfrentar el problema de las lesiones y la violencia en la región.

Palabras clave: seguridad; comunidad; lesiones; violencia; prevención; promoción de la salud
Fandiño-Losada A, Bangdiwala SI, Gutiérrez MI, Svanström L. Safe communities: a synopsis.

Salud Publica Mex 2008;50 suppl I:S78-S85.

\begin{abstract}
The "Safe Community" is a community-based intervention model by the World Health Organization (WHO) for control and prevention of injuries and violence. The program is directed towards the prevention of all types of injuries and to all age groups, environments and situations. In this model, the community identifies its organizational needs, defines interest areas with participation by everybody, designs and implements interventions by means of inter-sector work using local, low cost solutions, and guarantees the sustainability of the programs by means of governmental commitment and alliances among different organizations. This model has demonstrated its effectiveness in different contexts, including developing countries. In Latin America, a "Safe Community" has been certified in Chile, an example to be followed for facing the problem of injuries and violence in the region.
\end{abstract}

Key words: safety; community; injuries; violence; prevention; health promotion

(I) Social Medicine, Department of Public Health Sciences, Karolinska Institutet, Stockholm, Sweden. Investigador Asociado, Instituto CISALVA, Universidad del Valle. Cali, Colombia.

(2) Research Professor, Department of Biostatistics, School of Public Health, University of North Carolina at Chapel Hill, NC, USA. Visiting Professor, Department of Public Health Sciences, Karolinska Institutet. Stockholm, Sweden.

(3) Directora, Instituto CISALVA, Universidad del Valle, Cali, Colombia. Profesora Titular, Escuela de Salud Pública, Universidad del Valle. Cali, Colombia.

(4) Chair WHO Collaborating Centre on Community Safety Promotion at Karolinska Institutet. Chair/ Professor of Social Medicine, Department of Public Health Sciences, Karolinska Institutet. Stockholm, Sweden.

Fecha de recibido: 31 de julio de 2007 • Fecha de aprobado: 7 de noviembre de 2007 Solicitud de sobretiros: Leif Svanström, PhD. Social Medicine, Norrbacka, Plan 2. SE 17176 Stockholm, Sweden. Correo electrónico: Leif.Svanstrom@ki.se 
L a Comunidad Segura es un modelo de intervención de la Organización Mundial de la Salud (OMS) para el control y la prevención de las lesiones y la violencia basado en la comunidad. ${ }^{1}$ Su característica fundamental es la creación de una infraestructura local para la prevención de las lesiones y la promoción de la seguridad. Este abordaje promueve la cooperación entre los diferentes actores de una comunidad, entre ellos las agencias gubernamentales, el sector privado, las organizaciones comunitarias y los miembros de la sociedad civil en general.

El modelo de las Comunidades Seguras de la OMS es un enfoque que se ha incorporado y aplicado en el campo de la salud pública en los últimos 30 años alrededor del mundo. Tiene sus orígenes en un proyecto piloto para la prevención de las lesiones en Falköping, Suecia, en $1975,{ }^{2}$ y ha evolucionado hasta alcanzar el desarrollo y certificación de 115 comunidades seguras en todos los continentes en este momento, ${ }^{3}$ las cuales cuentan con poblaciones que oscilan entre 2000 y 2000000 habitantes.

Los programas de promoción de la seguridad están orientados a prevenir todos los tipos de lesiones y dirigidos a todos los grupos de edad, ambientes y situaciones, con la colaboración de diferentes actores locales. Las intervenciones son múltiples (educativas, ambientales, legislativas, etc.) y se evalúan con los datos de los sistemas de información disponibles en la comunidad para realizar los ajustes necesarios y continuar su funcionamiento.

\section{Definición de conceptos}

La prevención de las lesiones y la promoción de la seguridad son campos de acción de la salud pública relativamente recientes. Todavía existen muchas confusiones en relación con la definición de seguridad y se han propuesto conceptos tan variados como las actividades para la prevención del crimen y la violencia, el sentimiento subjetivo de estar libre de todo tipo de peligro o la satisfacción de todas las necesidades básicas. No todas estas definiciones se vinculan con las lesiones de causa externa o su prevención. Para unificar conceptos, la OMS y sus centros colaboradores para la promoción de la seguridad y la prevención de las lesiones han propuesto un marco conceptual y operacional conocido como "Documento de Quebec", ${ }^{4}$ que incluye las siguientes definiciones:

1. Seguridad. Es un estado en el cual los riesgos y las condiciones que generan un daño físico, psicológico o material se controlan para preservar la salud y el bienestar de los individuos y la comunidad. La seguridad es un recurso esencial para la vida diaria y necesario para que los individuos y las comunidades alcancen sus aspiraciones y expectativas; ${ }^{4}$ se trata de un proceso dinámico y no tan sólo de la ausencia de lesiones o amenazas. ${ }^{5}$ De manera adicional, la seguridad es una necesidad fundamental de los seres humanos, al igual que las necesidades fisiológicas. ${ }^{6} \mathrm{El}$ "Manifiesto de Estocolmo para las comunidades" seguras estableció que "la seguridad es un derecho humano", tal y como los otros derechos humanos de la Declaración de las Naciones Unidas; en consecuencia, también debe promoverse y protegerse. ${ }^{7}$

2. Promoción de la seguridad. Es el proceso para desarrollar y mantener la seguridad y lo aplican los individuos, las comunidades, los gobiernos y otras organizaciones, en los planos local, nacional e internacional. Incluye todos los esfuerzos realizados para la modificación de las estructuras, los ambientes (físico, social, tecnológico, político, económico y organizacional), las actitudes y los comportamientos relacionados con la seguridad. ${ }^{4}$ El concepto de promoción de la seguridad es mucho más amplio que el de prevención de las lesiones, de la misma forma que el concepto de salud es mucho más extenso que la mera ausencia de enfermedad. ${ }^{8}$

3. Comunidad. Existen dos conceptos de comunidad, el primero se refiere a las personas que habitan en una localización geográfica específica (pueblo, ciudad, municipalidad, etc.) y el segundo a una entidad ligada a las interacciones y lazos sociales que las personas construyen entre sí (asociaciones profesionales, grupos de interés, organizaciones comunitarias, etc.). Estos términos no son excluyentes entre sí y el sentido de comunidad se construye a partir de los dos. ${ }^{9,10}$

4. Comunidad segura. Una comunidad segura puede ser una municipalidad, condado, ciudad o distrito (comuna) de una ciudad que trabaje en la promoción de la seguridad y la prevención de las lesiones, los desastres naturales, el suicidio y la violencia, con programas orientados a todos los grupos de edad y género, en todas las áreas, y que sea parte de una red internacional de programas acreditados. ${ }^{1}$

Para mayor información sobre los conceptos de promoción de la seguridad y las comunidades seguras, consúltese el sitio en internet del Centro Colaborador de la OMS para la Promoción Comunitaria de la Seguridad del Instituto Karolinska de Suecia.*

\footnotetext{
* http://www.phs.ki.se/csp/who_publications_en.htm.
} 


\section{Desarrollo histórico}

En la década de 1960 surgió un interés creciente por el trabajo comunitario. El primer programa orientado hacia la salud se enfocó en la prevención de enfermedades mentales en Skövde, Suecia, ${ }^{8}$ y en el decenio de 1970 se desarrollaron programas comunitarios de prevención de las enfermedades coronarias. ${ }^{11,12}$ Respecto a las políticas públicas, las raíces del movimiento de comunidades seguras pueden rastrearse hasta los conceptos de La Nueva Salud Pública y la Salud para Todos de la OMS, y más tarde en la "Carta de Ottawa para la promoción de la salud" ${ }^{13}$ Esta última define campos de actividad relacionados con las comunidades seguras: la creación y aplicación de políticas públicas saludables, la creación de ambientes seguros y el fortalecimiento de la acción comunitaria. Los principios de la "Carta de Ottawa" también se han aplicado en otras iniciativas de base comunitaria para la promoción de la salud: Las Escuelas Saludables y Los Municipios Saludables. Estas iniciativas se desarrollaron en forma paralela al modelo de las comunidades seguras y son complementarias de este último, ya que se orientan a problemas de salud diferentes, como las enfermedades infecciosas, la nutrición y la contaminación ambiental, aunque en algunas experiencias también han abordado problemas relacionados con las lesiones y la violencia. ${ }^{14}$

Las enseñanzas del Programa de Prevención de Accidentes de Falköping (1978-1991) ${ }^{1}$ se aplicaron en Lindköping y Motala (Suecia) y también en Wang Khoi (Tailandia) para probar este abordaje en un contexto sociocultural completamente diferente. Producto de estas experiencias se diseñó la Guía para el Desarrollo de las Comunidades Seguras, ${ }^{15}$ la cual se explica más adelante. Los primeros programas se idearon para identificar lesiones no intencionales, a través de los sistemas de vigilancia de lesiones en los hospitales y sitios de atención primaria y en la década de 1980 se concedió gran énfasis a la prevención de la violencia y el crimen.

El concepto de comunidades seguras inició su existencia formal durante la Primera Conferencia Mundial de Prevención de Lesiones y Accidentes de 1989 en Estocolmo. Como producto de esta conferencia se suscribió el "Manifiesto por las Comunidades Seguras", 7 el cual declara que "Todos los seres humanos tienen el mismo derecho a la salud y la seguridad". De modo adicional, el Centro Colaborador de la OMS para la Promoción Comunitaria de la Seguridad del Instituto Karolinska, Suecia, recibió la responsabilidad de promover el modelo de las comunidades seguras de la OMS y establecer la Red Mundial de Comunidades Seguras para coordinar los esfuerzos para desarrollar, instituir y difundir esta metodología.*

\section{El abordaje de las comunidades seguras}

Esta iniciativa es diferente a otros programas de prevención de las lesiones porque la comunidad interviene de una manera activa y protagónica en la identificación y la solución de sus problemas de seguridad; para ello recurre a los abordajes de políticas públicas "arriba-abajo" y "abajo-arriba". Combinar estos métodos es la forma más efectiva de priorización e intervención, ya que emplea las organizaciones y sistemas gubernamentales que ya estén bien establecidos (arriba-abajo) y el conocimiento y las necesidades de los individuos de la comunidad (abajo-arriba). ${ }^{8}$ El término "comunidades seguras" implica que la comunidad aspira a lograr la seguridad mediante un abordaje estructurado de sus problemas, no que ésta sea un lugar perfectamente seguro. Los programas deben caracterizar de manera adecuada el problema existente y evaluar con regularidad la efectividad de las intervenciones instituidas.

El concepto de comunidades seguras debe tener en cuenta la estructura y organización de cada sociedad. Algunas sociedades están organizadas alrededor de un modelo local descentralizado de decisiones y en éstas es relativamente fácil desarrollar pequeños programas locales sin tener como referencia otros niveles de la sociedad, por lo menos en los estadios tempranos. Inevitablemente se descubren más tarde problemas que no pueden resolverse en el plano local y es necesario construir lazos con las áreas regional, nacional o aun internacional. ${ }^{16}$ En otras sociedades, la organización formal limita las iniciativas locales si no se logra con anterioridad una aprobación oficial desde los planos regional o central.

\section{Guía para el desarrollo de una comunidad segura}

Con base en las experiencias de Lidköping, Suecia, y Wang Khoi, Tailandia, se han desarrollado cinco principios básicos para suministrar un fundamento metodológico a los proyectos de comunidades seguras. ${ }^{15}$ Estos principios y sus componentes se explican a continuación:

\section{A. Necesidades organizativas para el trabajo}

* Véase http://www.phs.ki.se/csp/index_en.htm. 
de las comunidades seguras

1. El control de las lesiones en la comunidad debe apoyarse en todas las organizaciones de relevancia local, sin perder de vista las fortalezas y debilidades de cada organización.

2. El programa debe estar vinculado de forma estrecha con todos los sectores sociales relevantes, y en particular con el sector de la atención primaria en salud.

3. La estructura utilizada para promover el programa varía de una comunidad a otra y de un país a otro, pero debe aprovechar los procesos de decisiones más efectivos existentes en la comunidad y conceder apoyo a los mecanismos de decisión menos eficientes.

4. La comunidad participará en la solución de los problemas y proveerá algunos recursos para estas actividades, sólo si las personas reconocen como relevantes los beneficios de los programas de intervención propuestos.

\section{B. Fuentes de información necesarias para identificar} a los grupos y las áreas más vulnerables y difusión de la información sobre seguridad

1. La prevención comunitaria de las lesiones debe estar fundamentada en suficiente información epidemiológica y de otros tipos, para documentar la magnitud y la naturaleza del problema de las lesiones en todos los ambientes.

2. La comunidad debe reconocer sus posibilidades para la prevención y el control de las lesiones, además de la naturaleza de sus problemas de seguridad en el área local. Este objetivo se puede lograr si se utilizan los medios masivos de comunicación y cualquier otra forma de difusión de información que emplee la comunidad.

3. La difusión de la información debe ser relevante y acorde con las características de la audiencia; asimismo, debe presentarse de forma sencilla y hacer énfasis especial en los factores locales.

4. La información acerca de cómo prevenir y controlar las lesiones debe adaptarse a las condiciones locales de la comunidad.

\section{Intervención y participación, sus objetivos y fundamentos}

1. En general, la comunidad debe participar en las intervenciones que son de su interés.

2. La comunidad debe aceptar las intervenciones $y$, en la medida de lo posible, deben beneficiar a la mayor cantidad de personas.

3. Las soluciones deben ser aplicables a los procesos sociales, económicos y políticos existentes a corto plazo; empero, la continuidad de los programas debe garantizarse a largo plazo.

4. Las intervenciones deben estar fundamentadas en un abordaje intersectorial.

5. Los objetivos deben definirse con claridad. Deben realizarse mediciones de línea de base y una recolección adecuada de datos para que éstos sean útiles al evaluar los procesos y los resultados de las intervenciones realizadas.

\section{Lista de prioridades para las decisiones}

1. La priorización de las acciones debe basarse en los problemas que la comunidad identifica como los más importantes.

2. Las decisiones comunitarias deben tomarse a partir del reconocimiento de los problemas enfrentados y sus posibles soluciones (incluido el conocimiento de que muchas de estas soluciones son baratas).

3. La participación comunitaria debe comenzar desde el inicio del programa. La comunidad no debe adoptar soluciones que imponga un agente externo.

4. La intervención debe procurar obtener un éxito temprano susceptible de medición, que estimule a la comunidad a emprender más acciones.

5. En cuanto sea posible, la comunidad debe sugerir las soluciones. No deben imponerse soluciones procedentes de otros lugares, sino que éstas deben adaptarse, si bien sólo si las percibe como apropiadas la comunidad.

6. Los conocimientos relevantes sobre posibles soluciones e intervenciones deben presentarse a la comunidad de manera simple y clara.

\section{E. Tecnologías, métodos y recursos para instituir} los programas e intervenciones

1. Es necesario que surja una conciencia del problema en los planos gubernamental y comunitario. Para ello es necesario usar un amplio rango de técnicas, incluidos los medios masivos, la presentación local de datos, los programas en las escuelas y las visitas personales a quienes toman las decisiones.

2. La recolección de datos sobre la violencia y las lesiones debe ser sencilla, sólo lo suficiente para mostrar el patrón de los sucesos que conducen a las lesiones e identificar los lugares peligrosos, los 
grupos de riesgo y los productos dañinos. El alcance de la recolección de datos debe mantenerse dentro de los recursos y la tecnología inmediatamente disponibles.

3. La comunidad debe recibir apoyo del proceso de desarrollo comunitario, de manera que éste opere con libertad en la identificación de los peligros y el hallazgo de soluciones aceptables en el plano local. La asistencia técnica debe estar disponible de modo permanente para que actúe como catalizadora de los procesos y provea la información técnica requerida.

4. Debe usarse un amplio espectro de métodos para identificar los peligros en la comunidad, incluidas las rondas de seguridad, las listas de revisión, los hallazgos de investigaciones de otros lugares y los estímulos para que las personas notifiquen situaciones peligrosas. Los receptores de esta información pueden variar de un proyecto a otro.

5. Los recursos deben obtenerse mediante la participación y el trabajo de la comunidad, no sólo a través de su contribución económica. En algunos países es posible obtener fondos de los recursos públicos y el patrocinio de las empresas privadas. El uso simultáneo de financiación gubernamental para complementar los fondos locales actúa como un incentivo. Cuando sea posible, la comunidad decide sobre la forma de conseguir fondos para ofrecer sostenibilidad a los programas de prevención de las lesiones y la violencia.

6. Las comunidades tienen la responsabilidad de influir en las políticas gubernamentales y las prácticas de las compañías privadas en los temas que alteran la seguridad de las personas. Los gobiernos asumen la responsabilidad de legislar y desarrollar los procesos de decisiones que complementen los esfuerzos de las personas para lograr el objetivo de ser una comunidad segura.

7. Los métodos que modifican el ambiente físico frente a peligros o que logran la protección automática (pasiva) de los individuos son vitales si se quiere obtener la mayor reducción de las lesiones. Por lo general, estos métodos son sencillos y de bajo costo. Los proyectos deben tratar de inventar nuevas tecnologías que ofrezcan medidas de protección pasiva a bajo costo, por ejemplo formas de separar el tránsito de vehículos y peatones o la instalación de protectores para cubrir las partes en movimiento de las máquinas.

8. La comunidad debe promover el uso de equipos de seguridad, como la ropa protectora, los cascos, y los protectores de ojos. Sin embargo, debe garantizarse la selección de equipos de suficiente calidad, capaces de suministrar una protección real y adecuada; asimismo, debe evitarse y controlarse el uso de elementos de seguridad imperfectos o falsificados, los cuales confieren una falsa sensación de seguridad.

\section{Criterios de las comunidades seguras internacionales de la OMS}

A partir de 2002, las comunidades deben satisfacer seis criterios para ser designadas como una Comunidad Segura internacional de la OMS que se describen en el cuadro I. ${ }^{1}$

\section{Evaluación de la efectividad del programa de comunidades seguras}

Los programas de intervención como las comunidades seguras se pueden clasificar en el campo denominado Investigación Participativa de Base Comunitaria. ${ }^{17}$ Esta metodología se fundamenta en un proceso iterativo de actividades, reflexiones y aprendizaje basado en experiencias y difiere de los diseños de investigación experimentales en lo siguiente:

1. No es práctico tener un grupo de comunidades que sirvan de grupo control para comparar los efectos de la intervención.

2. No es posible asignar las comunidades de manera aleatoria a la intervención y enmascararlas; por lo tanto, no se pueden evitar los posibles sesgos del proceso de selección y asignación de las intervenciones.

3. Las intervenciones, por naturaleza, son muy dife-

\section{Cuadro I \\ LOS SEIS CRITERIOS DE LAS COMUNIDADES SEGURAS INTERNACIONALES DE LA OMS}

En el escenario de las comunidades seguras, las comunidades deben tener:

I. Una infraestructura basada en colaboraciones y asociaciones, gobernada por un grupo representante de varios sectores encargado de la promoción de la seguridad en la comunidad.

2. Programas sostenibles a largo plazo, que incluyan a ambos géneros y todas las edades, ambientes y situaciones.

3. Programas cuyos objetivos sean los grupos y ambientes de alto riesgo, y programas que promuevan la seguridad de los grupos vulnerables.

4. Programas que documenten la frecuencia y las causas de las lesiones y la violencia.

5. Medidas de evaluación para valorar los programas, los procesos y los efectos del cambio.

6. Participación continua en las redes nacionales e internacionales de las comunidades seguras. 
rentes entre las comunidades y por consiguiente no hay uniformidad en el tipo de intervención a evaluar.

Estos programas de intervención se consideran como diseños cuasiexperimentales y su evaluación debe tomar en cuenta aspectos cuantitativos y cualitativos. Una evaluación tiene en esencia dos objetivos: juzgar si la intervención es efectiva en reducir el riesgo de lesiones y determinar si la intervención debe continuarse, modificarse o descartarse. ${ }^{18}$ Este último objetivo incluye consideraciones de índole práctica y económica y aspectos no mensurables del beneficio de un programa en una comunidad.

La evaluación cualitativa se basa en un análisis descriptivo de los procesos de la intervención. Se efectúan observaciones sobre aspectos de la organización, la comunicación, las actividades realizadas, la participación de quienes toman decisiones, la cobertura poblacional y la institución y uso de la intervención.

Para el análisis cuantitativo se deben decidir los criterios de evaluación a medirse, los cuales pueden ser indicadores de salud, económicos o sociales. En un estudio controlado, la efectividad se mide como la diferencia entre el grupo intervenido y el grupo sin intervención. En los estudios "antes-después", la efectividad se cuantifica como la diferencia entre el periodo posterior y el periodo anterior a la intervención. ${ }^{19}$ Para evaluar múltiples programas se requiere el uso de modelos probabilísticos, como las series de tiempo, los estudios de panel (o longitudinales) y los modelos de datos de multinivel, según sean la naturaleza de los datos disponibles y la existencia de grupos de control. ${ }^{20}$ También se pueden utilizar las técnicas de revisiones sistemáticas y los metaanálisis.

\section{Resultado de la evaluación del modelo de las comunidades seguras de la OMS}

Spinks, Turner, Nixon y McClure utilizaron en fecha reciente los criterios ya descritos para evaluar el modelo de las comunidades seguras de la OMS, ${ }^{21}$ pero encontraron limitaciones metodológicas, como la diversidad de las intervenciones y la falta de detalles sobre las actividades específicas de las intervenciones. Este hecho limitó el número de publicaciones y comunidades en la revisión y tampoco permitió consolidar de forma estadística los resultados de las revisiones (metaanálisis). A pesar de estas deficiencias metodológicas, los autores concluyeron que existen evidencias que sugieren que el modelo de las comunidades seguras de la OMS es efectivo para reducir las lesiones en las comunidades que lo han aplicado.

\section{Discusión}

\section{Las comunidades seguras en Latinoamérica y EI Caribe}

Según cifras del Proyecto de Carga Mundial de la Enfermedad de la OMS para el año 2000, en la región de Latinoamérica y El Caribe, se presentaban $7.82 \%$ de la mortalidad y $7.95 \%$ de la carga de enfermedad producidas por las lesiones de causa externa en todo el mundo. ${ }^{22}$ A pesar de la magnitud del problema en la región, se han documentado muy pocas experiencias comunitarias de prevención de las lesiones. Al realizar una búsqueda en las bases de datos PubMed ${ }^{23}$ y SciELO, ${ }^{24}$ a partir de las palabras claves "comunidad", "prevención", "lesiones", "traumas", "accidentes" y "violencia" para Latinoamérica y El Caribe, sólo se encontraron seis referencias relacionadas con programas para la prevención de lesiones de tránsito, de la violencia y las lesiones en niños, en Brasil, Colombia, Ecuador, Jamaica y México. 25-30

Para ensombrecer aún más este panorama, las lesiones y la violencia no han merecido la atención suficiente por parte de los gobiernos, las organizaciones sociales y la academia, hecho que señaló Blank en su editorial para Injury Prevention: "El control de las lesiones para un gran número de personas en las Américas continúa en el siglo $X X$, mientras que la pandemia [de las lesiones] muestra una faceta [propia] del siglo $\mathrm{XXI}$. ${ }^{31}$

El Modelo de las Comunidades Seguras de la OMS ha demostrado ser una medida efectiva para la prevención de las lesiones y la violencia en las comunidades en las cuales se ha instituido. Esta metodología está en funcionamiento en contextos diversos, como los países nórdicos (Suecia, Noruega, Dinamarca, Finlandia) con un alto nivel de desarrollo y naciones en desarrollo como Sudáfrica, Vietnam e Irán, con características culturales muy diferentes entre sí. ${ }^{1}$ Aunque la mayoría de las comunidades seguras pertenece a países desarrollados, esta metodología tiene en cuenta la limitada disponibilidad de recursos en las comunidades de los países en desarrollo.

Este modelo se ha establecido en Europa, Norteamérica, Asia, África y Oceanía, pero hasta el año pasado no se había designado ninguna comunidad segura en la región de Latinoamérica y El Caribe. Ahora la situación ha cambiado y este año se han producido tres hitos en el desarrollo del movimiento de las comunidades seguras en la región:

1. La municipalidad de Peñaflor, en Chile, ha sido 
designada como la primera comunidad segura de Latinoamérica. Su programa de promoción de la seguridad funciona desde el año 2001 y está dirigido a todos los grupos de edad, focalizado en la prevención de lesiones en el trabajo, el transporte, el hogar, la escuela y la recreación, además de la prevención de la violencia. El programa se dirige desde la alcaldía y cuenta con la participación de múltiples actores comunitarios.,**

2. El Instituto CISALVA de la Universidad del Valle, en Cali, Colombia, ha sido designado como el primer Centro Certificador de Comunidades Seguras en el continente americano. Un centro certificador, además de la función que le da su nombre, tiene la misión de apoyar y promover el desarrollo de las comunidades seguras en la región, evaluar su trabajo y contribuir al conocimiento científico sobre la promoción comunitaria de la seguridad.

3. La traducción al español del libro Safety Promotion - An Introduction de los doctores Welander, Svanström y Ekman. Este libro, que se publicará en fecha próxima, explica los conceptos teóricos y la aplicación práctica de la promoción de la seguridad, incluido el abordaje de las comunidades seguras.

\section{Conclusiones}

Las lesiones de causa externa y la violencia son un importante problema de salud pública en Latinoamérica y El Caribe que afecta de manera predominante a los grupos más jóvenes de la población. El modelo de las comunidades seguras de la OMS prioriza y promueve la búsqueda de soluciones locales de bajo costo para enfrentar esta problemática y posibilita su institución y sostenimiento a largo plazo en las comunidades de los países en desarrollo. Tan sólo se requiere el compromiso de trabajar de manera coordinada por una comunidad más segura por parte de los diferentes sectores y actores de la sociedad: desde el gobierno local hasta las organizaciones de base y los ciudadanos.

La municipalidad de Peñaflor, en Chile, es un ejemplo a seguir dentro de esta iniciativa para la promoción de la seguridad en las comunidades de Latinoamérica y El Caribe. En este momento, otras comunidades de Bolivia, Colombia, Ecuador y Perú preparan sus nominaciones como comunidades seguras de la OMS, con el apoyo del Instituto CISALVA como centro certi-

\footnotetext{
* Véase http://www.phs.ki.se/csp/safecom/penaflorchile.htm y http:// www.municipalidadpeñaflor.cl/

‡ Véase http://www.cisalva.univalle.edu.co/.
}

ficador.

Ésta es una invitación para unir esfuerzos con el movimiento de las comunidades seguras de la OMS para hacer de las municipalidades, ciudades y distritos de Latinoamérica y El Caribe lugares más agradables y seguros.

\section{Referencias}

I.WHO Collaborative Centre on Community Safety Promotion at the Karolinska Institutet. The safe community network (monograph). Stockholm: Karolinska Institutet, 1999:6-13.

2. Schelp L. Epidemiology as a basis for evaluation of a community intervention programme on accidents (thesis). Stockholm: Karolinska Institutet, 1987:42-64.

3.WHO Collaborative Centre on Community Safety Promotion at the Karolinska Institutet. Safe communities network members 2007 [consultado I 2 julio 2007]. Disponible en http://www.phs.ki.se/csp/who_ safe_communities_network_en.htm.

4. Maurice P, Lavoie M, Charron RL, Chapdelaine A, Bonneau HB, Svanström, et al. Safety and safety promotion: conceptual and operational aspects. Québec: Centre collaborateur OMS du Québec pour la promotion de la sécurité et la prévention des traumatismes, Réseau de santé publique et Ministère de la Santé et des Services sociaux du Québec,WHO Collaborating Centre on Community Safety Promotion at Karolinska Institutet, Sweden and World Health Organisation, 1998: I - 19.

5. Svanström L. Evidence-based injury prevention and safety promotion

- some results. In: Samhällets olycksbarn, Förslag till nationella jämlikhetsstrategier - flera skadefria år för alla (report). Stockholm: Sweden's National Institute of Public Health, 1998.

6. Maslow AH. Toward a psychology of being. NewYork:VanNostrand, 1968. 7.World Health Organisation. Manifesto for Safe Communities. Proceedings of the Ist World Conference on Accident and Injury Prevention, Stockholm. Geneva:WHO, 1989.

8. Welander G, Svanström L, Ekman R. Community safety promotion - Safe Communities. In: Safety promotion - an introduction. $2 \mathrm{~d}$ ed. Stockholm: Karolinska Institutet, 2004:97-I24.

9. Heller K.The return to community. Am J Community Psychol 1989;।7:I-15.

10. McMillan DW. Sense of community.J Community Psychol 1996;24(4):315-325.

I I. Puska P, Nissinen A, Tuomilehto J, Salonen JT, Koskela K, McAliister A, et al.The community-based strategy to prevent coronary heart disease: conclusions from the ten years of the North Karelia project. Ann Rev Public Health 1985;6:147-193.

12. Nilsen P.The theory of community based health and safety programs: a critical examination. Inj Prev 2006; I2: |40-|45.

13. World Health Organization. Ottawa charter for health promotion. First International Conference on Health Promotion Ottawa, 2 I November 1986 - WHO/HPR/HEP/95.I. (report). Ottawa:WHO, 1986. 14. Organización Panamericana de la Salud. Municipios, ciudades y comunidades saludables: recomendaciones para la evaluación dirigidas a los responsables de las políticas en las Américas. Washington, DC: Organización Panamericana de la Salud, 2005.

15. Moller J, Havanonda S, Romer C, Svanström L. Formulating guidelines for safe communities (report). Sundbyberg: Karolinska Institutet, 1989:2-31. 16. Havanonda S. Safe community. Bangkok: United Publication Press, 1991. 17. Faridi Z, Grunbaum JA, Gray BS, Franks A, Simoes E. Communitybased participatory research: necessary next steps. Prev Chronic Dis 2007;4(3):A70.

18. Doll L, Bartenfeld T, Binder S. Evaluation of interventions designed to 
prevent and control injuries. Epidemiol Rev 2003;25:5 I-59.

19. Hauer E. Observational before-after studies in road safety. Estimating the effect of highway and traffic engineering measures on road safety. Oxford: Pergamon Press, 1997.

20. Bangdiwala SI. Statistical considerations for the design, conduct and analysis of the efficacy of safe community interventions. Int J Inj Contr Saf Promot 2001;8:91-97.

21. Spinks A, Turner C, Nixon J, McClure R. The 'WHO Safe Communities' model for the prevention of injury in whole populations (review).

Cochrane Database Syst Rev 2005;(2):CD004445.

22. Murray CJL, Lopez AD, editors. The global burden of disease. Boston: Harvard University Press, 1996:613-687.

23. National Library of Medicine/ National Instititutes of Health. PubMed [consultado 12 julio 2007]. Disponible en: http://www.ncbi.nlm.nih. gov/sites/entrez

24. Scientific Electronic Library Online. SciELO. [consultado 12 julio 2007]. Disponible en: http://www.scielo.org/index.php?lang=es.

25. Liberatti CLB,Andrade SM, Soares DA. The new brazilian traffic code and some characteristics of victims in southern Brazil. Inj Prev 2001;7:190-193.

26. Rodríguez JM, Muñoz E, Fandiño-Losada A, Gutiérrez MI. Evaluación de la estrategia de comunicación "Mejor hablemos" para promover la convivencia pacífica en Cali, 1996-2000. Rev Salud Publica 2006;8(3): I68-184.

27. Gutiérrez-Martínez MI, Espinosa-Del Vallín R, Fandiño A, Oliver RL.The evaluation of a surveillance system for violent and non-intentional injury mortality in Colombia cities. Int J Inj Contr Saf Promot 2007;14(2):77-84. 28. Maira G. La violencia intrafamiliar: experiencia ecuatoriana en la formulación de políticas de atención en el sector salud. Rev Panam Salud Publica 1999;5(4/5):332-337.

29. Sacco FC, Twemlow SW. School violence reduction: a model Jamaican secondary school program. Community Ment Health J 1997;33(3): 229-234. 30. Mock C,Arreola-Risa C, Trevino-Pérez R,Almazan-Saavedra V, Zozaya-Paz JE, González-Solis R, et al. Injury Prevention conselling to improve safety practices by parents in Mexico. Bull World Health Organ 2003;8I(8):59I-598.

31. Blank D. Injury control in South America: the art and science of disentanglement. Inj Prev 2004;10:321-324. 